TRANSACTIONS OF THE

AMERICAN MATHEMATICAL SOCIETY

Volume 250, June 1979

\title{
WEAK CUTS OF COMBINATORIAL GEOMETRIES
}

\author{
BY
}

\author{
HIEN Q. NGUYEN
}

\begin{abstract}
A weak cut of a Combinatorial Geometry $G$ is a generalization of a modular cut, corresponding to the family of the new dependent sets in a weak map image of $G$.

The use of weak cuts allows the construction of all weak images of $G$, an important result being that, to any family $\pi$ of independent sets of $G$, is associated a unique weak cut $\mathcal{C}$ containing $\mathfrak{T}$. In practice, the flats of the weak image defined by $\mathcal{C}$ can be constructed directly. The weak cuts corresponding to known weak maps, such as truncation, projection, elementary quotient, are determined.

The notion of weak cut is particularly useful in the study of erections. Given a geometry $F$ and a weak image $G$, an $F$-erection of $G$ is an erection of $G$ which is a weak image of $F$. The main results are that the set of all $F$-erections of $G$ is a lattice with the weak map order, and that the free $F$-erection can be constructed explicitly. Finally, a problem involving higher order erection is solved.
\end{abstract}

Introduction. In the theory of Combinatorial Geometries, whereas strong maps have been widely studied, much fewer research papers have focused on the more general concept of weak maps. After the pioneering work of Higgs [5], [6] on maps of geometries, Lucas' thesis [9] was probably the first study to concentrate specially on problems concerning weak maps. In addition to their intrinsic interest, the study of weak maps is highly relevant to several important questions and open conjectures in the general theory of Combinatorial Geometries, as is well shown in [9]. It can also be noted that examples of weak maps abound in algebraic geometry: for example, consider the specialization of a point in general position in 3-space onto a plane or a straight line. In this paper, we consider the problem of constructing weak map images and preimages of a given geometry, with the view of developing a technique useful for analyzing problems related to weak maps in general.

A fundamental procedure in the study of strong maps is to use the one-to-one correspondence between strong maps and modular cuts. The main approach in this paper is to introduce a more general notion of cuts, called weak cuts, for the case of weak maps.

Received by the editors July 25, 1977 and, in revised form, April 14, 1978.

AMS (MOS) subject classifications (1970). Primary 05B25, 05B35; Secondary 05A20, 06A20.

Key words and phrases. Combinatorial geometry, combinatorial pregeometry, matroid, weak map, independent system, erection. 
$\S 1$ is devoted to a short review of relevant definitions and notations. In $\$ 2$, weak cuts are introduced and a first application is to construct all weak maps of a given geometry $G$. An important result is that any family $\mathfrak{N}$ of independent sets of $G$ defines uniquely a weak map image of $G$ in which they become dependent. This weak image is said to be generated by $\mathfrak{N}$ and its flats can be constructed directly from $\mathfrak{T}$. In $\$ 3$, well-known weak maps, such as truncation, projection, elementary quotient, are shown to be easily defined in terms of weak cuts. $\$ 4$ considers weak map preimages of $G$, and special attention is given to those preimages whose truncations coincide with $G$, i.e. erections of $G$. In fact, a more general situation is studied, namely dominated erections, corresponding to the erections of $G$ which are themselves weak images of another given geometry $F$. The use of weak cuts not only provides a simple proof of a generalization of Crapo's result [2], namely that the set of erections of $G$ dominated by $F$ is a lattice with the weak order, but also gives a simple construction of the freest element in this lattice, the analog of the free erection. In the case of higher order erections, the notion of weak cuts has also been helpful in giving the insight for answering a question posed by Las Vergnas in [8].

1. Definitions and notations. Throughout this paper, $S$ will denote a finite set. $\mathscr{P}$ is the power set of $S$ and $\mathscr{P}_{n}$ its subfamily consisting of all subsets with $n$ elements, called $n$-subsets. An independence family $\mathcal{G}$ is a subset of $\mathscr{P}$ satisfying the following two properties:

(1) $\mathcal{G}$ is an order ideal of $\mathscr{P}$ (i.e. if $I \in \mathcal{G}$ and $J \subset I$ then $J \in \mathscr{G}$ ).

(2) If $I, J \in \mathcal{G}$ and $|I|=|J|+1$, then there exists an element $x \in I$ such that $J \cup\{x\} \in g$.

The subfamily $\mathscr{G} \cap \mathscr{P}_{n}$ will be denoted by $\mathscr{G}_{n}$. An independence family $\mathscr{G}$ defines a (combinatorial) geometry $G(G)$ on $S$ (also known as combinatorial pregeometry in [3] or matroid). When there is no ambiguity, $G(\Phi)$ will be simply written $G$. A subset $I \subset S$ is independent in $G$ if $I \in \mathcal{G}$ and dependent otherwise. For $A \subset S$, a basis of $A$ is a maximal independent set contained in $A$. A fundamental property of combinatorial geometries is that all bases of $A$ have the same cardinality, $r_{G}(A)$, the rank of $A$. The rank function $r_{G}$ satisfies the semimodularity property: for $A, B \subset S, r_{G}(A)+r_{G}(B) \geqslant r_{G}(A \cup B)+$ $r_{G}(A \cap B) . r_{G}(S)$ is the rank of the geometry. The closure of $A \subset S$ in $G$ is written $\bar{A}^{G}$, and $\bar{A}^{G}=\{x \mid x \in S, r(A \cup\{x\})=r(A)\}$. A flat $F$ of $G$ is a closed set, i.e. $F=\bar{F}^{G}$. A circuit is a minimal dependent set.

Given two geometries $G$ and $H$ on $S$, corresponding to two independence families $g_{G}$ and $g_{H}$, the notation $G \rightarrow H$ indicates that there is a weak map from $G$ to $H$, i.e. there is a permutation $\pi$ on $S$ such that for any independent set $I$ in $H, \pi^{-1}(I)$ is independent in $G$. Without loss of generality (see [9] for example) we will assume in this paper, that $\pi$ is in fact the identity on $S$, so 
that the notation $G \rightarrow H$ is equivalent to $\mathscr{G}_{H} \subset \mathscr{G}_{G} . H$ is then a weak image of $G$, and $G$ is a weak preimage of $H$. The set inclusion order among independence families induces an order on all geometries on $S$, called the weak map order: the largest (or freest) elements is the geometry corresponding to $\mathscr{P}$, called the Boolean Algebra on $S$ and denoted by $B$; the smallest is the geometry of rank 0 defined by $\varnothing$. If $G$ covers $H$ in this order, the map $G \rightarrow H$ is simple. The map $G \rightarrow H$ is strong if any closed set in $H$ is also closed in $G$. $H$ is then a quotient of $G . H$ is an elementary quotient if $r_{H}(S)=r_{G}(S)-1$.

For a detailed account of the theory of combinatorial geometries, the reader is referred to [3].

The following simplified set notation will be used: for $A \subset S, a \in S$, $A \cup a, A-a$, etc. will stand for $A \cup\{a\}, A-\{a\}$, etc. The symbol indicates that either a proof is completed or that a trivial proof is omitted.

2. Weak cuts. Given a geometry $G$ on $S$, the action of a weak map on $G$ is to add dependence among points of $G$, i.e. transform some independent sets of $G$ into dependent sets of the new geometry. Weak images of $G$ can thus be constructed by specifying the families of new dependent sets. The main motivation of this paper is to study the structure of these families and develop a useful approach to analyze problems related to weak maps.

Let $\mathcal{G}$ and $G^{\prime}$ be the independence families corresponding to $G$ and a weak image $H$ of $G$ : we have $g^{\prime} \subset 9$. We observe the following facts:

(1) $g-g^{\prime}$ is the family of sets which are independent in $G$ but dependent in $H$;

(2) If $A$ is an independent set in $G$ which is dependent in $H$, then any independent set in $G$ containing $A$ is also dependent in $H$;

(3) If $A$ is an independent set in $H$, and $x, y$ are elements of $S$ such that $A \cup x$ and $A \cup y$ are dependent in $H$ (they may be independent or not in $G)$, then we have: $r_{H}(A \cup x \cup y)=r_{H}(A)=|A|$. Consequently, any set of cardinality $|A|+1$ contained in $A \cup x \cup y$ is dependent in $H$, in particular those which are independent in $G$.

It turns out that the last two preceding remarks are also sufficient to determine a weak image of $G$. More precisely, we define the structure of weak cuts as follows.

Definition 2.1. Given a geometry $G$ on $S$ defined by the independence family $G$, a weak cut $C$ of $G$ is a subset of $G$ satisfying the following properties:

W1. $\mathcal{C}$ is an order filter of $\mathscr{G}$ (i.e. if $I \in \mathcal{C}$ and $I \subset J \in \mathcal{G}$, then $J \in \mathcal{C}$ ).

W2. For $I \in G-\mathcal{C},|I|=n$, and $x, y \in S$, if $I \cup x$ and $I \cup y$ do not belong to $G-C$, then any element of $\mathscr{G}_{n+1}$ contained in $I \cup x \cup y$ belongs to $e$.

The following fundamental result indicates the relationship between weak 
cuts and weak maps:

Proposition 2.2. Given a geometry $G$ defined by the independence family $G$, a subset $G^{\prime}$ of $G$ is an independence family if and only if $G-G^{\prime}$ is a weak cut of G.

Proof. Suppose that $G^{\prime} \subset \mathcal{G}$ is an independence family defining a weak image $H$ of $G$ and let $\mathcal{C}=\mathscr{G}-\mathscr{G}^{\prime}$. Clearly, $\mathcal{C}$ is the family of sets which are independent in $G$ but dependent in $H$. By our previous remarks, $C$ satisfies properties $\mathrm{W} 1$ and $\mathrm{W} 2$.

In order to prove the sufficiency part of Proposition 2.2, we have to show that, if $C$ is a subset of $G$ satisfying $W 1$ and $W 2$, then $G-C$ is an independence family. This will be a consequence of the following lemmas.

LEMMA 2.3. $\mathcal{C}$ being a subset of 9 satisfying $\mathrm{W} 1$, if $I \in \mathcal{G}-\mathcal{C}$ and $J \subset I$, then $J \in G-\mathcal{C}$.

LEMMA 2.4. If $\mathcal{C}$ is a subset of $\mathcal{G}$ satisfying $\mathrm{W} 1$ and $\mathrm{W} 2$, then $\mathcal{C}$ also satisfies:

W3. For $I \in \mathcal{G}-\mathcal{C},|I|=n$, and $x_{1}, x_{2}, \ldots, x_{p} \in S$, if $I \cup x_{i}$ does not belong to $\mathcal{G}-\mathcal{C}, 1 \leqslant i \leqslant p$, then any element of $\mathcal{G}_{n+1}$ contained in $I \cup x_{1} \cup$ $x_{2} \cup \cdots \cup x_{p}$ belongs to $\circlearrowright$.

Proof. The proof is by induction on $p$. For $p=2$, W3 is identical to W2. Assume that $\mathrm{W} 3$ is satisfied for any $p, 2 \leqslant p \leqslant q-1$, and let us consider the case $p=q$.

If W3 is not satisfied, there is an element $X$ of $g_{n+1}$, a subset of $I \cup x_{1} \cup$ $x_{2} \cup \ldots \cup x_{q}$, such that $X \in G-\mathcal{G}$. Because of the induction hypothesis, namely that any element of $g_{n+1}$ contained in $I \cup x_{i_{1}} \cup \ldots \cup x_{i_{q-1}}$ belongs to $C$,we have $\left\{x_{1}, x_{2}, \ldots, x_{q}\right\} \subset X$. Let us write $X=I_{1} \cup x_{1} \cup \ldots \cup x_{q}$ where $I_{1} \subset I$, and consider $Y=I_{1} \cup x_{1} \cup \ldots \cup x_{q-2} \cdot|Y|=|X|-2=|I|$ $-1=n-1$.

Claim. There exists an element $a$ of $I-I_{1}$ such that $Y \cup a$ belongs to $9-$ e.

Proof. Suppose that this is not the case, and let $I-I_{1}=\left\{a_{1}, a_{2}, \ldots, a_{s}\right\}$. As $\left|I_{1}\right|+s=|I|=|X|-1=\left|I_{1}\right|+q-1$, we have $s=q-1$. As $X \in g$ $-\mathcal{C}$, and $Y \subset X$, by Lemma 2.3, we $Y \in G-\mathcal{C}$. Now, by assumption that the claim does not hold, for any $a_{i}, 1 \leqslant i \leqslant s, Y \cup a_{i} \notin 9-\mathcal{G}$. As $s=q-$ 1 , the induction hypothesis can be invoked, i.e. W3 holds, to infer that any element of $G_{n}$ contained in $Y \cup a_{1} \cup \ldots \cup a_{s}$ belongs to $\mathcal{C}$. This is a contradiction as $I \subset Y \cup a_{1} \cup \ldots \cup a_{s}$ and $I \in g-\mathcal{C}$. The claim is thus proved.

We can now proceed with the proof of Lemma 2.4. There is an element $a$ of $I-I_{1}$, such that $Y \cup a=I_{1} \cup a \cup x_{1} \cup \ldots \cup x_{q-2}$ belongs to $q-C$. $|Y \cup a|=|I|=n$. Now, the set $Y \cup a \cup x_{q-1}$ is a subset of cardinality 


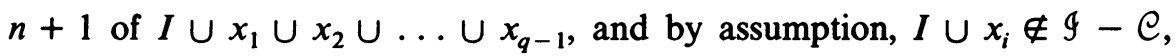
for $1 \leqslant i \leqslant q-1$. If $Y \cup a \cup x_{q-1}$ is independent, i.e. $Y \cup a \cup x_{q-1} \in$ $q_{n+1}$, then by induction hypothesis, i.e. that W3 holds for $p<q-1$, we have $Y \cup a \cup x_{q-1} \notin 9-\mathcal{C}$. If $Y \cup a \cup x_{q-1}$ is dependent, then $Y \cup a \cup x_{q-1}$ $\notin \mathcal{G}$, and finally in all cases, we can say that $Y \cup a \cup x_{q-1} \notin \mathcal{G}-\mathcal{C}$. For the same reasons, $Y \cup a \cup x_{q} \notin q-\mathcal{C}$. Then by W2, any element of $q_{n+1}$ contained in $(Y \cup a) \cup x_{q-1} \cup x_{q}$ belongs to $C$, in particular $X$, which is a contradiction. This completes the proof of Lemma 2.4 .

LEMMA 2.5. For $I \subset A \subset S, I \in \mathcal{G}_{n}-\mathcal{C}$, if any element of $\mathscr{G}_{n+1}$ contained in $A$ belongs to $\mathcal{C}$, then any element of $\Phi_{n+1}$ contained in $A \cup \bar{I}^{G}$ also belongs to $\mathrm{C}$.

Proof. This is a direct consequence of Lemma 2.4, because for any element $x$ of $\left(A \cup \bar{I}^{G}\right)-I$, we have $I \cup x \notin G-\mathcal{C}$.

We now proceed to prove the sufficiency part of Proposition 2.2. Let $\mathcal{C}$ be a weak cut. We need to show that $g^{\prime}=G-C$ is an independence family.

By Lemma 2.3, $g^{\prime}$ is an order ideal.

Let $I, J \in g^{\prime}$ and $|I|=|J|+1=n$. $I$ and $J$ being independent in $G$, let $K=\{x \mid x \in I, J \cup x \in 9\}$. We have $K \neq \varnothing$ and $I-K \subset \bar{J}^{G}$. Suppose that for any $x \in K, J \cup x \in \mathcal{C}$. By Lemmas 2.4 and 2.5, any element of $g_{n}$

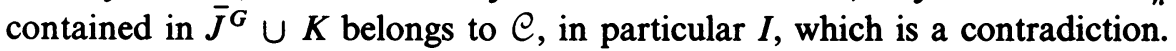
Thus for some $x \in K, J \cup x \in G^{\prime}$ and $g^{\prime}$ is an independence family.

By Proposition 2.2, to each weak map of $G$ corresponds a unique weak cut and conversely. It is thus equivalent to study weak maps or weak cuts. For example, it is clear that if $\mathcal{C}_{1}$ and $\mathcal{C}_{2}$ are two weak cuts corresponding to two weak maps $G \rightarrow H_{1}$ and $G \rightarrow H_{2}$, we have $H_{1} \rightarrow H_{2}$ if and only if $\mathcal{C}_{1} \subset \mathcal{C}_{2}$ : characterizing simple weak maps of $G$ corresponds to characterizing minimal weak cuts of $G$.

The main application of Proposition 2.2 in this paper is to develop a construction of all weak cuts of $G$. Considered as a subset of $\mathcal{G}$, any weak cut is an order filter. As is the case with many order filters, it may seem natural to try to define a weak cut $C$ by saying that $C$ is the minimal subset of $g$ satisfying $\mathrm{W} 1, \mathrm{~W} 2$ and containing a certain given family $\Re$ of independent sets of $G$. Such an approach turns out not to be possible because there are in general many minimal subsets of 9 containing $\Re$ which satisfy W1 and W2: for example, if $G$ is the geometry depicted in Figure 1 (below), then the two geometries depicted in Figures 2 and 3 correspond to two minimal weak cuts of $G$ containing $\{45,235\}$. In other words, the intersection of two weak cuts is not a weak cut in general. This is exactly what has made the question of completely describing the action of weak maps difficult (see [11] for example). 
Proposition 2.2 yields a constructive characterization of weak cuts which we now proceed to describe.

The following equivalent forms of $\mathrm{W} 1$ and $\mathrm{W} 2$ will be useful.

Proposition 2.6. $C$ being a subset of $\mathscr{G}$ and $C_{n}=\bigodot \cap g_{n}$, $e$ is a weak cut of $G$ if and only if $\mathcal{C}_{i}$ satisfies the following properties for any integer $i$, $0<1<|S|:$

$\mathrm{W}_{i}$. If $I \in \mathcal{G}_{i}$ and, for some $x \in I, I-x \in \mathcal{C}_{i-1}$, then $I \in \mathcal{C}_{i}$.

$\mathrm{W} 2_{i}$. For $I \in g_{i-1}-C_{i-1}$ and $x, y \in S$, if $I \cup x$ and $I \cup y$ do not belong to $G_{i}-C_{i}$, then any element of $G_{i}$ contained in $I \cup x \cup y$ belongs to $C_{i}$.

Given a subset $\mathfrak{N}$ of $\mathscr{G}$, we construct inductively a weak cut $\mathcal{C}$ containing $\Re$ as follows:

$e_{0}=\varnothing$.

For $i \geqslant 1, C_{i}=\bigodot \cap g_{i}$ is the smallest subset of $g_{i}$ satisfying properties $\mathrm{Wl}_{i}$ and $\mathrm{W} 2_{i}$ containing the elements of $\Re$ of cardinality $i$.

In order to show that the construction is valid, we need the following lemma which guarantees that for $i \geqslant 1$, each $C_{i}$ is uniquely defined.

Lemma 2.7. For $i \geqslant 1$, given $\mathcal{C}_{i-1}$ and $M$, subsets of $\Phi_{i-1}$ and $\Phi_{i}$ respectively, there is a smallest subset $\mathcal{C}_{i}$ of $G_{i}$ satisfying $\mathrm{W}_{1}$ and $\mathrm{W}_{i}$, and containing $M$.

Proof. We first note that $g_{i}$ does satisfy $\mathrm{W}_{i}, \mathrm{~W} 2_{i}$ and contains $C$. Let $M_{1}$ and $M_{2}$ be two subsets of $\Phi_{i}$ satisfying $\mathrm{W}_{i}, \mathrm{~W} 2_{i}$ and containing $M$ : it is clear that $M_{1} \cap M_{2}$ also has the same properties. $C_{i}$ can thus be defined as the intersection of all subsets of $g_{i}$ satisfying $\mathrm{W}_{i}, \mathrm{~W}{ }_{i}$ and containing $C: \mathcal{C}_{i}$ is always defined and uniquely so.

In practice, in order to construct $C_{i}$, one may start with (1) the elements of $\Re$ of cardinality $i$ and (2) the elements $I$ of $g_{i}$ such that for some $x \in I$, $I-x \in \mathcal{C}_{i-1}$, and then add the elements of $\mathscr{G}_{i}$ as needed in order to satisfy property W2 ${ }_{i}$. In this fashion, the first subset of $g_{i}$ which satisfies $\mathrm{W} 2_{i}$ will be $e_{i}$.

The efficiency or practicality of the construction depends on how fast $\mathcal{C}_{i}$ is completed, i.e. how many times $\mathrm{W} 2_{i}$ has to be invoked during the construction. From this practical point of view, Lemmas 2.4 and 2.5 are more useful than W2, because they correspond to several repeated applications of W2. It may also be worthwhile to obtain a stronger and more "efficient" version of Lemma 2.5. Let $H$ be the weak image of $G$ corresponding to $C$. The conditions listed in the statement of Lemma 2.5 concerning $I$ and $A$ are equivalent to saying that $r_{H}(A)=|I|=i-1$, so that no $i$-subset contained in $\bar{A}^{H}$ can be independent in $H$, in particular the elements of $G_{i}$ contained in $A \cup \bar{I}^{G}$. A stronger version will consist in saying that under the same conditions, any element of $G_{i}$ contained in some subset of $\bar{A}^{H}$, strictly larger 
than $A \cup \bar{I}^{G}$, also belongs to $\bigodot_{i}$. Such a set is the following: let $c(A)$ denote the smallest subset of $S$ containing $A$ such that, if it contains $J \in \mathcal{G}-\mathcal{C}$, then it also contains $\bar{J}^{G}$. It is clear that $c(A)$ is uniquely defined and that $A \cup \bar{I}^{G} \subset c(A) \subset \bar{A}^{H}$.

ExAMPLE. Let $G$ be the geometry represented in Figure 1, where all the lines containing more than two points are indicated.

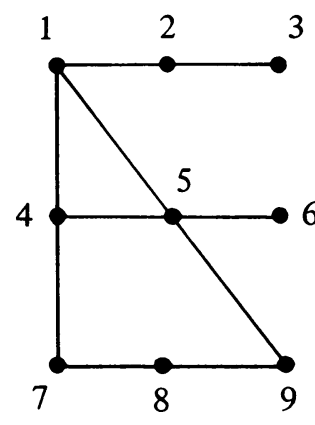

Figure 1

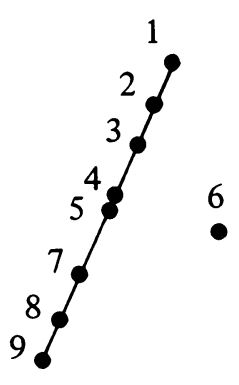

FIgURE 2

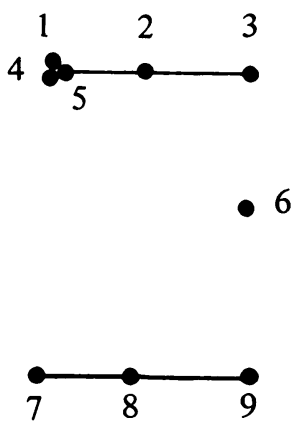

FIgURE 3

Let $\Re$ consist of the two independent sets 45 and 235. Proceeding inductively, we have

$$
\bigodot_{0}=\varnothing, \quad e_{1}=\varnothing, \quad \varrho_{2}=\{45\}
$$

$e_{3}$ contains 451, 452, 453, 457, 458, 459 and 235.

As 453,235 belong to $C_{3}$ and 35 to $\Phi_{2}-C_{2}$, we have $234 \in C_{3}$ by $W 2_{3}$.

Next, as $234 \in C_{3}$ and $23 \in g_{2}-C_{2}$, any element of $g_{3}$ contained in $c(234)=12345789$ also belongs to $C_{3}$.

The family $\left\{I \mid I \in \mathscr{G}_{3}, I \subset 12345789\right\}$ satisfies $\mathrm{W} 2_{3}$, so it is equal to $\mathcal{C}_{3}$.

As $G_{i}=\varnothing$ for $i \geqslant 4$, we have $\mathcal{C}=\cup_{0<i<3} \mathcal{C}_{i}$ defining the weak image depicted in Figure 2.

By the construction described above, any subset $\mathscr{R}$ of $\mathcal{G}$ defines a unique weak cut, called the weak cut generated by $\mathfrak{N}$. Conversely any weak cut $\mathcal{C}$ is generated by some subset of 9 :

Proposition 2.8. Any weak cut $\mathcal{C}$ is generated by its minimal elements.

Proof. Let $\mathfrak{T}$ be the set of minimal elements of $\mathcal{C}$. We want to show that $\mathcal{C}^{\prime}$, the weak cut generated by $\mathfrak{N}$, coincides with $\mathcal{C}$. Let us use the notation

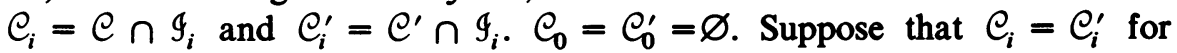
$0 \leqslant i \leqslant k-1$ and consider the case $i=k$. If an element $I$ of $\bigcup_{k}$ is minimal in $\mathcal{C}$, then it belongs to $\mathcal{C}_{k}^{\prime}$ by construction. If $I \in \mathcal{C}_{k}$ is not minimal, it has a subset belonging to $\mathcal{C}_{k-1}=\mathcal{C}_{k-1}^{\prime}$, so $I$ also belongs to $\mathcal{C}_{k}^{\prime}$. Thus $\mathcal{C}_{k} \subset \mathcal{C}_{k}^{\prime}$. On 
the other hand, $e_{k}$ clearly satisfies $W 2_{k}$. As, by construction, $\mathcal{C}_{k}^{\prime}$ is the smallest subset of $g_{k}$ containing $e_{k}$ satisfying $\mathrm{W} 2_{k}$, we have $e_{k}^{\prime} \subset C_{k}$. Finally, $\mathcal{C}_{k}=\mathcal{C}_{k}^{\prime}$.

A similar argument can show that, if $\mathcal{C}$ is the weak cut generated by $\Re \subset \mathcal{G}$, then $\mathcal{C}$ is a minimal weak cut containing $\Re$, i.e. if $\mathcal{C}^{\prime}$ is a cut such that $\mathfrak{R} \subset \mathcal{C}^{\prime} \subset \mathcal{C}$, then $\mathcal{C}^{\prime}=\mathcal{C}$. There are however other minimal cuts containing $\mathfrak{T}$, in general. For example, the weak cut of $G$ depicted in Figure 1 , generated by $\mathfrak{T}^{\prime}=\{14,15\}$, is another minimal weak cut containing $\Re=\{45,235\}$. The corresponding weak image is represented in Figure 3.

Whereas any subset $\mathfrak{T}$ of $\mathscr{G}$ generates a weak cut uniquely, a weak cut may be generated by many different subsets of 9 . In the previous example, the weak cut $\mathcal{C}$ generated by $\mathfrak{N}$ can also be generated by any of the following as well: $\{45,234\}\{45,359\},\{45,234,237,278\}$, etc . ...

To any subset $\mathfrak{N}$ of $\mathscr{G}$, is associated a unique weak image $H$ of $G$. The independent sets of $H$ are easily determined from the weak cut generated by $\Re$. One may wonder whether it is possible to obtain other cryptomorphic definitions of $H$, e.g. in terms of rank-function, flats, etc ..., directly from $\Re$. It turns out that the flats of $H$ can be constructed from $\Re$ as follows: we will determine inductively the closure operator of $H$ denoted by $C$ and the family of independent sets of $H$ denoted by $\mathcal{H}$.

Let $C(\varnothing)=\{x \mid x \in S,\{x\} \in \mathscr{N}\}$ and $\mathcal{H}_{0}=\varnothing$.

For $i>0$, let

$$
\mathscr{H}_{i}=\left\{I \mid I \in \mathscr{G}_{i}, \forall j<i, \forall J \in \mathscr{H}_{j}, I \not \subset C(J)\right\} .
$$

To each $I \in \mathcal{H}_{i}$ we associate a subset $C(I)$ of $S$ such that the family $\left\{C(I) \mid I \in \mathcal{H}_{i}\right\}$ satisfies the following properties:

F1. For $I \in \mathcal{H}_{i}, \bar{I}^{G} \subset C(I)$.

F2. If $J \in \mathcal{H}_{j}, j<i$ and $J \subset C(I)$, then $C(J) \subset C(I)$ if $j<i$ and $C(J)=C(I)$ if $j=i$.

F3. For $M \in \mathfrak{R},|M|=i+1$, if $C(I) \cap M \in \mathcal{F}_{i}$ then $M \subset C(I)$.

F4. $\left\{C(I) \mid I \in \mathcal{F}_{i}\right\}$ is minimal with properties $\mathrm{F} 1, \mathrm{~F} 2$ and $\mathrm{F} 3$, in the sense that, if $\left\{C^{\prime}(I) \mid I \in \mathcal{F}_{i}\right\}$ is any other family satisfying $F 1, F 2$ and $F 3$, then $C(I) \subset C^{\prime}(I)$.

First we note that if two families $\left\{C_{1}(I) \mid I \in \mathcal{H}_{i}\right\}$ and $\left\{C_{2}(I) \mid I \in \mathcal{H}_{i}\right\}$ satisfy F1, F2 and F3, then the intersection family $\left\{C_{1}(I) \cap C_{2}(I) \mid I \in \mathcal{H}_{i}\right\}$ also satisfies $F 1, F 2$ and $F 3$. On the other hand, the family $\{S\}$, i.e. any $I \in \mathcal{H}_{i}$ is associated to $S$, does satisfy $\mathrm{F} 1, \mathrm{~F} 2$ and $\mathrm{F} 3$. The family $C(I)$ is thus the intersection of all families of subsets of $S$ satisfying F1, F2 and F3: it always exists and is unique.

Proposition 2.9. For $i \geqslant 0, \mathcal{H}_{i}$ is the family of independent sets of $H$ with cardinality $i$, and for $I \in \mathcal{H}_{i}, C(I)$ is the closure of $I$ in $H$. 
Proof. Proposition 2.9 is true for $i=0$. Let us assume that it is true for $0 \leqslant i \leqslant p-1<r_{H}(S)$ and consider the case $i=p$. For any set $J$ independent in $H,|J| \leqslant p-1, C(J)$ is thus the closure of $J$ in $H$, and also, any flat of $H$ of rank $r<p-1$ is of the form $C(J)$ for some $J \in \mathcal{H}_{r}$.

It is clear that $\mathcal{K}_{p}$ is the family of independent sets of $H$ with cardinality $p$. For $I \in \mathcal{H}_{p}$, we have to show that $C(I)=\bar{I}^{H}$. Consider the family $\left\{\bar{I}^{H} \mid I \in\right.$ $\left.\mathcal{H}_{p}\right\}$ : it satisfies F1 $\left(\bar{I}^{G} \subset \bar{I}^{H}\right), \mathrm{F} 2$ (if $J \in \mathcal{H}_{j}, j<p, J \subset \bar{I}^{H}$, then $\bar{J}^{H} \subset \bar{I}^{H}$ if $j<p$, and $\bar{J}^{H}=\bar{I}^{H}$ if $\left.j=p\right)$ and F3 (M is dependent in $H$, and $r_{H}\left(\bar{I}^{H} \cap\right.$ $M)=p=|M|-1$, so that $\left.M \subset \overline{I^{H}} \cap M^{H}=\bar{I}^{H}\right)$. As $\left\{C(I) \mid I \in \mathcal{H}_{i}\right\}$ satisfies $\mathrm{F} 4$ we have $C(I) \subset \bar{I}^{H}$.

In order to complete the proof of Proposition 2.9, the following lemma is needed.

LEMMA 2.10. $\mathcal{C}$ being the weak cut generated by $\mathfrak{N}$, any element $X$ of $\mathcal{C}_{p+1}$ is contained in some $C(J)$ for $J \in \mathcal{H}_{p}$.

Proof. Case 1. $X=M \in \mathscr{T},|M|=p+1 . M$ is dependent in $H: r_{H}(M)$ $<p$. Let $A$ be a basis of $M$ in $H$. If $|A|=p$, then $M \subset C(A)$ by $\mathrm{F} 3$ and $A \in \mathcal{H}_{p}$. If $|A|<p$, then by induction hypothesis $C(A)=\bar{A}^{H}$ and $M \subset \bar{A}^{H}$. As $r_{H}(S) \geqslant p$, it is possible to complete $A$ into an independent set $J$ in $H$ of rank $p$, so that $M \subset \bar{A}^{H}=C(A) \subset C(J)$.

Case 2. $X \in \mathcal{G}_{p+1}$ such that for any $y \in X, X-y \in \mathcal{C}_{p}$. As $X-y \notin \mathcal{H}_{p}$, $X-y \subset C\left(J_{1}\right)$ for some $J_{1} \in \mathcal{H}_{j}, j<p$. Let $J_{2}=J_{1} \cup y$ if $J_{1} \cup y$ is independent in $H$, and $J_{2}=J_{1}$ otherwise. $J_{2}$ can be completed into an independent set $J_{3}$ of $H$ of rank $p$, and we have $X \subset C\left(J_{3}\right)$.

General case. In general, in the construction of $\mathcal{C}_{p+1}$ in order to satisfy property $\mathrm{W} 2_{p+1}$, elements of $\oint_{p+1}$ are added to the initial family consisting of the subsets $X$ considered in Cases 1 and 2 above. Each of these subsets is contained in some $C(J)$ for $J \in \mathcal{H}_{p}$. Suppose that $Y$ is the first element of $g_{p+1}$ added to form $C_{p+1}$, that is not contained in any $C(J)$, for $J \in \mathcal{H}_{p}$. As this is by application of $\mathrm{W} 2_{p+1}$, we have $Y \subset J \cup y \cup z$, for some $J \in \mathscr{G}_{p}-$ $\mathcal{C}=\mathcal{H}_{p}$ and $y, z \in S$ are such that $J \cup y, J \cup z \notin G-\mathcal{C}$. There are three possibilities:

$\cdot J \cup y, J \cup z \in \mathcal{C}_{p+1}$, i.e. both sets are independent in $G$. Then, by hypothesis, $J \cup y \subset C\left(J_{1}\right)$ and $J \cup z \subset C\left(J_{2}\right)$ for some $J_{1}$ and $J_{2} \in \mathcal{H}_{p}$. Because of $\mathrm{F} 2$, we have $C(J)=C\left(J_{1}\right)=C\left(J_{2}\right)$, so that $Y \subset J \cup y \cup z \subset$ $C(J)$, which is a contradiction.

$\cdot J \cup y \in \mathscr{G}_{p+1}$ and $J \cup z \notin \mathscr{G}_{p+1}$. Then, $J \cup y \subset C\left(J_{1}\right)$ and $z \in \bar{J}^{G} \subset$ $C(J) \subset C\left(J_{1}\right)$, so that again $Y \subset C(J)$, which is a contradiction.

- Both $J \cup y$ and $J \cup z$ are dependent in $G$. Then, $y, z \in \bar{J}^{G} \subset C(J)$ so that $Y \subset C(J)$.

The proof of Lemma 2.10 is thus complete. 
We can now proceed with the proof of Proposition 2.9 by showing that $\bar{I}^{H} \subset C(I)$. Let $x \in \bar{I}^{H}$ : there is a circuit $K$ in $H$, such that $x \in K$ and $K \subset I \cup x$. If $|K|<p+1$, then $x \in \overline{K-x^{H}}=C(K-x) \subset C(I)$. Suppose that $|K|=p+1$, i.e. $K=I \cup x$. If $I \cup x$ is dependent in $G, I$ being independent, then $x \in \bar{I}^{G} \subset C(I)$. If $I \cup x$ is independent in $G$, then $I \cup x \in \mathcal{C}_{p+1}$. By Lemma 2.10, $I \cup x$ is contained in some $C(J)$ for $J \in$ $\mathcal{H}_{p}$, and by $\mathrm{F} 2, C(J)=C(I)$. Thus $I \cup x \subset C(I)$. Finally, in all cases, we have $x \in C(I)$, so that $\bar{I}^{H} \subset C(I)$.

Proposition 2.9 justifies the following construction of the flats of the weak image generated by $\mathscr{T}$. Proceeding inductively, set $\mathcal{K}_{0}=\varnothing$ and $C(\varnothing)=$ $\{x \mid\{x\} \in \mathscr{N}\}$. For $i>0, \mathcal{H}_{i}$ is defined as $\left\{I \mid I \in \mathscr{G}_{i}, \forall j<i, \forall J \in \mathcal{H}_{j}\right.$, $I \not \subset C(J)\}$. The family of flats of rank $i$ is obtained by starting with the family $\left\{\bar{I}^{G} \mid I \in \mathcal{F}_{i}\right\}$ and progressively enlarging its elements by repeated applications of F2 or F3, until the family satisfies F2 and F3. This idea of constructing geometric flats by starting with "seeds" and then enlarging these in order to satisfy specific necessary conditions has been used in other instances [7], [8], [10], for different purposes, and besides being rather natural, it is practical enough for computer experimentation [7].

EXAMPle. Consider $G$ depicted in Figure 1 and $\Re=\{45,235\}$.

(a) $i=0: \mathscr{K}_{0}=\varnothing, C(\varnothing)=\varnothing$.

(b) $i=1: \mathcal{H}_{1}$ consists of all singletons of $\mathscr{P}$. To construct the corresponding flats, initialize with $\{1,2,3,4,5,6,7,8,9\}$ which gives $\{1,2,3,4,5,6,7,8,9\}$ by $F 3$. This last family satisfies $F 2$ and F3.

(c) $i=2: \mathscr{H}_{2}$ consists of all pairs except 45. Initialize with $\{123,147,159,16,18,24,25,26,27,28,29,34,35,36,37$,

$$
38,39,456,48,49,57,58,59,67,68,69,789\} \text {. }
$$

By application of F3, we get the next family:

$\{12345,1457,1459,16,18,245,26,27,28,29,2345,36,37$,

$$
38,39,456,458,459,457,67,68,69,789\} \text {. }
$$

Applying F2 several times, we have:

$$
12345,1457,1459 \subset C(14) \Rightarrow 1234579 \subset C(14)
$$

and

$$
1234579,789 \subset C(79) \Rightarrow 12345789 \subset C(79) .
$$

Finally the family obtained thus far: $\{12345789,16,26,36,456,67,68,69\}$ satisfies F2 and F3.

(d) $i=3$ : As $G$ is of rank 3, its weak images are of rank at most 3, so the only flat of rank 3 is $S$ itself.

\section{Special cases.}

3.1. Weak cuts of a Boolean Algebra. As any geometry on $S$ can be considered as a weak image of $B$, the Boolean Algebra on $S$, Definition 2.1 
and Proposition 2.2 provide a set of axioms of combinatorial geometries, equivalent to other known cryptomorphic definitions.

$G$ is now the Boolean Algebra. A weak cut $\mathcal{C}$ being a subset of 9 consisting of the new dependent set in the weak image, in this case, $C$ is the set of all dependent sets of the weak image. We thus have the following.

Proposition 3.1.1. A family $\mathcal{C}$ of subsets of a finite set $S$ is the family of dependent sets of a geometry on $S$ if and only if $\mathcal{C}$ satisfies the following properties:

(1) $\mathcal{C}$ is an order filter.

(2) For $I \subset S,|I|=n, x, y \in S, I \notin \mathcal{C}$, if $\mathcal{C}$ contains $I \cup x$ and $I \cup y$, then $\mathcal{C}$ contains all $(n+1)$-subsets of $I \cup x \cup y$.

3.2. TRUnCATION. Let $G$ be a geometry of rank $n$ and with independence family 9 . The truncation of $G$ is the geometry $H$ of rank $n-1$ whose independent sets are those of $G$ in ranks $0,1, \ldots, n-1 . H$ is a weak image of $G$ and the corresponding weak cut is $Q_{n}$.

More generally, for $k \leqslant n$, the $k$-truncation of $G$ is the geometry of rank $n-k$ whose independent sets are those of $G$ in ranks $0,1, \ldots, n-k$. The $k$-truncation corresponds to the weak cut $\cup_{n-k+1<i<n} \Phi_{i}$.

3.3. Projection. Projections in geometries were introduced in [11] as generalizations of truncations. A $k$-truncation can be visualized as the "projection in general position" of the whole geometry on a flat of rank $n-k$. A projection $P_{F}(X)$ in a geometry $G$ is, informally, the projection of a subset $X$ of $S$ on a flat $F$ of $G$, preserving the dependence relationship of other points of $G$ as much as possible.

More formally, projections in $G$ are weak maps of $G$, and as such, they can be defined in terms of weak cuts. Given a subset $X$ of $S$ and a flat $F$ of $G$, if $A$ is a basis of $F$, the projection $P_{F}(X)$ is the weak image of $G$ corresponding to the weak cut generated by the family $\{A \cup x \mid x \in X, A \cup x$ independent in $G$ \}. It is not difficult to verify that this definition is equivalent to the one given in [11], where $P_{F}(X)$ is characterized by its flats obtained by a special case of the construction based on Proposition 2.9.

3.4. ElEMENTARY QUOTIENTS. Given a geometry $G$ on $S$ with independence family $\mathcal{G}$, a modular cut $\mathscr{F}$ [3] (modular filter in [4]), is a family of subsets of $S$ satisfying the following properties:

(1) $\mathcal{F}$ is an order filter.

(2) If $A, B$ are a modular pair in $G$ (i.e. $r_{G}(A)+r_{G}(B)=r_{G}(A \cup B)+$ $\left.r_{G}(A \cap B)\right)$ and $A, B \in \mathcal{F}$, then $A \cap B \in \mathcal{F}$.

The notion of modular cut is fundamental in the theory of strong maps: each modular cut determines a unique elementary quotient and conversely. More precisely, we have the following: 
Proposition [3], [4]. If $\mathscr{F}$ is a modular cut of $G, \mathcal{F}$ defines an elementary quotient $H$ whose rank-function satisfies: for $A \subset S$,

$$
\begin{aligned}
& r_{H}(A)=r_{G}(A)-1 \quad \text { if } A \in \mathscr{F}, \\
& r_{H}(A)=r_{G}(A) \quad \text { if } A \notin \mathscr{F} . \square
\end{aligned}
$$

An elementary quotient being a special weak map, the geometry $H$ can be specified by the corresponding weak cut, i.e. the set of new dependent sets $\left\{I\left|I \subset S, r_{H}(I)<r_{G}(I)=\right| I \mid\right\}$. It is clear that this weak cut is precisely $\mathscr{F} \cap \mathcal{G}$.

Proposition 3.4.1. If $\mathcal{F}$ is a modular cut of $G$, the elementary quotient corresponding to $\mathscr{F}$ is the weak image of $G$ determined by the weak cut $\mathscr{F} \cap 9$.

4. Weak preimages. Erections. A weak preimage of a geometry $G$ is a geometry $H$ such that $H \rightarrow G$. In greater generality, let $F$ and $G$ be two given geometries such that $F \rightarrow G$; we say that a geometry $H$ is a preimage of $G$ dominated by $F$ if $F \rightarrow H \rightarrow G$. As $H$ and $G$ are weak images of $F$, they are determined by two weak cuts $\mathcal{H}$ and $\mathcal{G}$ of $F$. As $H \rightarrow G$, we have $\mathcal{H} \subset \mathcal{G}$. Clearly, there is a one-to-one correspondence between the set of preimages of $G$ dominated by $F$ and the set of weak cuts of $F$ contained in $\mathcal{G}$. Weak preimages can thus be constructed using the basic method described in $\$ 2$.

In the remaining part of this section, we will consider the special class of erections.

$G$ being a given geometry, a geometry $H$ is a nontrivial erection of $G$ if $G$ is the truncation of $H$. The trivial erection of $G$ is $G$ itself. More generally, $F$ being a given weak preimage of $G$, we say that $H$ is an erection of $G$ dominated by $F$, or an $F$-erection of $G$, if $H$ is an erection of $G$ and $F \rightarrow H \rightarrow G$.

Let $\mathcal{G}$ be the weak cut of $F$ corresponding to $G$, and $\mathcal{G}$ the independence family of $F$. If the rank of $G$ is $n$, then for $i \geqslant n+1, \mathcal{G}_{i}=\mathscr{G}_{i}$. If $H$ is any $F$-erection of $G$, corresponding to a weak cut $\mathcal{H}$ of $F$, for $i \neq n+1$, $\mathcal{G}_{i}=\mathcal{H}_{i} . H$ is a nontrivial erection if and only if $\mathcal{G}_{n+1} \neq \mathcal{H}_{n+1}$. The weak cut $\mathcal{H}$ corresponding to an $F$-erection of $G$ will be called an erection cut and is completely determined by its subset $\mathcal{H}_{n+1}$. In this special case, Proposition 2.6 can be restated as:

Proposition 4.1. A subset $\mathcal{H}_{n+1}$ of $\mathscr{G}_{n+1}$ determines an erection cut $\mathcal{H}=(\mathcal{G}$ $\left.-\mathcal{G}_{n+1}\right) \cup \mathcal{H}_{n+1}$ if and only if:

(1) For $I \in 9_{n+1}$ and some $x \in I$, if $I-x$ is dependent in $G$, then $I \in$ $\mathcal{H}_{n+1}$.

(2) For $I$ a basis of $G$ and $x, y \in S$, if $I \cup x, I \cup y \notin \mathcal{G}-\mathcal{H}$ then $\mathcal{H}_{n+1}$ contains all $(n+1)$-subsets of $I \cup x \cup y$ independent in $F$. 
From Proposition 4.1, it is easy to verify that if $\mathcal{H}_{n+1}$ and $\mathcal{H}_{n+1}^{\prime}$ are two subsets of $\mathscr{G}_{n+1}$ satisfying the conditions listed, then so does $\mathcal{H}_{n+1} \cap \mathcal{H}_{n+1}^{\prime}$. Thus if $\mathcal{H}$ and $\mathcal{H}^{\prime}$ are two erection cuts, then $\mathcal{H} \cap \mathcal{H}^{\prime}$ is also an erection cut. The set of all erection cuts corresponding to $F$-erections of $G$, being closed under set-intersection, is a lattice. Because of the order antiisomorphism between erection cuts and $F$-erections, we have:

Proposition 4.2. Given two geometries $F$ and $G, F \rightarrow G$, the set of all $F$-erections of $G$, ordered by the weak map order, is a lattice.

The 1-element of the lattice of erection cuts corresponds to the cut determined by $\mathcal{H}_{n+1}=\mathscr{G}_{n+1}$, i.e. the trivial erection. The 0 -element is the cut defining the free $F$-erection of $G$. In the special case of $F$ being the Boolean Algebra, Proposition 4.2 is Crapo's well-known result [2]. We note that, not only is the proof of Proposition 4.2 significantly simpler than Crapo's original proof, but also it provides an easy construction of the free $F$-erection. Let $\mathcal{E}$ be the weak cut of $F$ generated by $\mathcal{G}-\mathcal{G}_{n+1}: \mathcal{E}$ is an erection cut. By

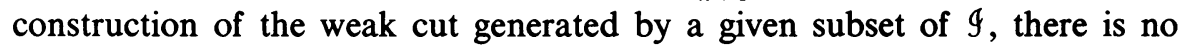
weak cut containing $\mathcal{G}-\mathcal{G}_{n+1}$ and properly contained in $\mathcal{E}$. $\mathcal{E}$ is thus the 0 -element of the lattice of erection cuts and determines a geometry $E$, the free $F$-erection. In order to construct $E$, it is sufficient to construct $\varepsilon_{n+1}$, the smallest subset of $g_{n+1}$ satisfying the conditions listed in Proposition 4. An element of $G_{n+1}$ is then a basis of the free erection if and only if it is not contained in $\varepsilon_{n+1}$. A geometry $G$ is not $F$-erectable (i.e. the only $F$-erection of $G$ is itself) if and only if the weak cut of $F$ generated by $\mathcal{G}-\mathcal{G}_{n+1}$ is $\mathcal{G}$ itself.

From a practical point of view, it is also possible to construct the flats of $E$, the free $F$-erection, using the algorithm based on Proposition 2.9. In this case, what is needed is the family of copoints of $E$, the other flats being the same as in $G$ and the independent sets of cardinality $n$ of $E$ being the bases of $G$.

Example. Let $F$ and $G$ be the geometries depicted in Figures 4 and 5 respectively, and $E$ the free $F$-erection of $G$.

The independent sets of cardinality 3 of $E$ are the bases of $G$. Using the method described we initialize with the family $\left\{\bar{I}^{F} \mid I\right.$ basis of $\left.G\right\}$ and enlarge its elements by repeated applications of rule F2 (in this case F3 is not used because $\mathfrak{T} \cap \mathscr{P}_{n+1}=\varnothing$ ). The important steps in the computation are as follows.

$$
\begin{aligned}
C(45) & =\overline{45}^{G}=456, C(37)=\overline{37}^{G}=378 . \\
456 & =C(45) \subset C(345) \Rightarrow 3456 \subset C(345) \text { and } \overline{456}^{F} \subset C(345) .
\end{aligned}
$$

Now, $\overline{456}^{F}=45678$ so that $345678 \subset C(345)$. 


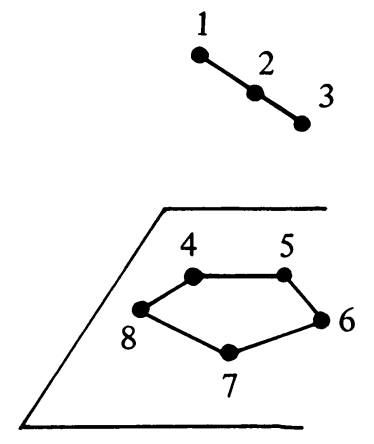

FIGURE 4

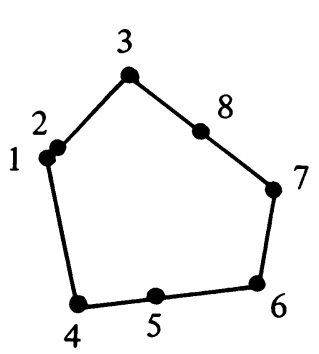

Figure 5

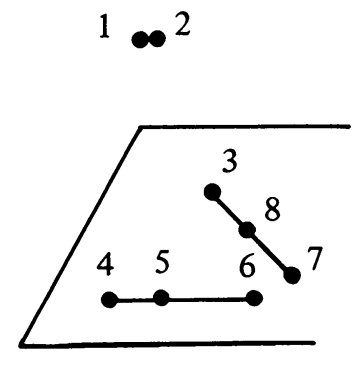

Figure 6

The final family obtained is $\{1234,1235,1236,12378,12456,1247,1248,1257,1258,1267,1268,345678\}$ corresponding to the geometry represented in Figure 6.

More generally, for $k \geqslant 1$ let a $k F$-erection of $G$ be a geometry $H$ whose $k$-truncation is $G$, and $F \rightarrow H \rightarrow G$. If $H$ is a $k F$-erection of $G$, the corresponding weak cut $\mathscr{H}$ of $F$ satisfies:

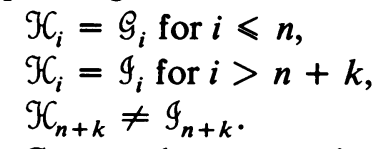

Conversely, any weak cut of $F$ with these properties defines a $k F$-erection of $G$ and will be called a $k$-erection cut. In particular, let $\delta$ be the weak cut of $F$ generated by $\cup_{1<i<n} \mathcal{G}_{i}$. If $\mathcal{E}_{n+k} \neq \mathscr{G}_{n+k}$ then the cut

$$
\left[\bigcup_{1<i<n+k} \mathcal{E}_{i}\right] \cup\left[\bigcup_{i>n+k} g_{i}\right]
$$

is a $k$-erection cut, say $\mathcal{E}^{k}$. If $E(G)$ denotes the free $F$-erection of $G$, the cut $\mathcal{E}^{k}$ defines $E^{k}(G)$, i.e. the free $F$-erection iterated $k$ times.

In this context, the following problems were posed in [8]: Given a geometry $H, G$ being the $k$-truncation of $H$, is it possible to erect $G$ freely $k$ times, i.e. does $E^{k}(G)$ exist? And if $E^{k}(G)$ exists, is $H$ a weak image of $E^{k}(G)$ ? Using the point of view of weak cuts, the problem can be reformulated as follows. Let $\mathcal{H}$ and $\mathcal{G}$ be the weak cuts of the Boolean Algebra $B$, corresponding to a geometry $H$ of rank $n+k$ and its $k$-truncation $G$. In the weak cut of $B$ generated by $\cup_{1<i<n} \mathcal{G}_{i}$, do we have $\mathcal{E}_{n+k}=\mathscr{P}_{n+k}$ ? And if not, is it true that for $n<i<n+k, \mathcal{E}_{i} \subset \mathcal{H}_{i}$ ?

As is general with weak cuts, the fact that $\mathcal{E}_{n+1} \subset \mathcal{K}_{n+1}$ does not guarantee any behavior for higher layers of the cuts: it may be that $\mathcal{E}_{n+k}=\mathscr{P}_{n+k}$ or, if not, that $\mathcal{K}_{n+k} \subset \mathcal{E}_{n+k}$. The following is an example of these assertions, 
showing that the answers to the questions asked above are negative.

Let $G$ be the geometry of rank 3 of Figure 7. All lines containing more than two points are indicated.

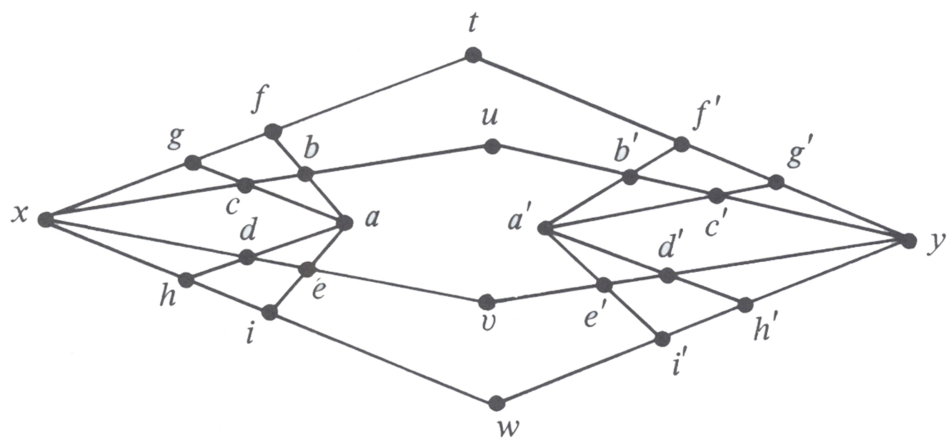

FIGURE 7

The free erection of $G$ is $E$, represented in Figure 8. We claim that $E$ is not erectable. Let $E^{\prime}$ be an erection of $E$. The set $\{a, x, t, u, v, w\}$ has rank 4 in $E^{\prime}$; so does $\left\{a^{\prime}, y, t, u, v, w\right\}$. Now, $\{t, u, v, w\}$ of rank 4 in $E$ is also of rank 4 in $E^{\prime}$, so that $\left\{a, a^{\prime}, x, y, t, u, v, w\right\}$ must be of rank 4 in $E^{\prime}$ : the whole geometry $E^{\prime}$ is of rank $4 . E^{\prime}$ is the trivial erection. Q.E.D.

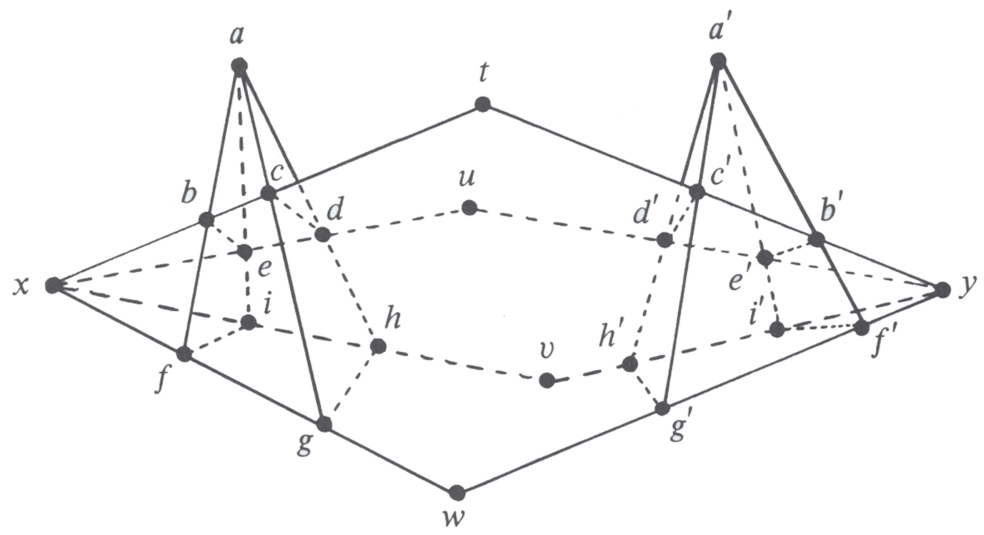

Figure 8

Consider now the geometry $H_{1}$ which differs from $E$ only by the fact that $r_{H_{1}}($ tuvw $)=3$. Clearly $H_{1}$ can be erected into a geometry $H_{2}$ of rank 5 , in which the set $\{x, y, t, u, v, w\}$ has rank 5 . In summary, we have found a geometry $\mathrm{H}_{2}$ such that its 2-truncation $G$ cannot be erected twice. 


\section{BIBLIOGRAPHY}

1. H. H. Crapo, Single element extensions of matroids, J. Res. Nat. Bur. Standards Sect. B 69 (1965), 55-65.

2. Erecting geometries, Proc. of the 2nd Chapel Hill Conference on Comb. Math. and Applications, Chapel Hill, 1970, 74-99.

3. H. H. Crapo and G. C. Rota, Combinatorial geometries, MIT Press, Cambridge, Mass., 1970.

4. T. A. Dowling and D. G. Kelly, Elementary strong maps between combinatorial geometries, Colloquio Internazionale sulle Teorie Combinatorie (Rome, 1973), Tomo II, Atti. Convegni Lincei, no. 17, Accad. Naz. Lincei, Rome, 1976, pp. 121-152.

5. D. A. Higgs, A lattice order on the set of all matroids on a set, Canad. Math. Bull. 9 (1966), 684-685.

6. __ Strong maps of geometries, J. Combinatorial Theory. Ser. 5 (1968), 185-191.

7. D. E. Knuth, Random matroids, Discrete Math. 12 (1975), 341-358.

8. M. Las Vergnas, On certain constructions for matroids, Proc. of the 5th British Combinatorial, 1975 , pp. $395-404$.

9. D. Lucas, Weak maps of combinatorial geometries, Trans. Amer. Math. Soc. 206 (1975), 247-279.

10. H. Q. Nguyen, Constructing the free erection of a combinatorial geometry, J. Combinatorial Theory Ser. B (to appear).

11. Projections and weak maps in combinatorial geometries, Research Notes No. 4, Department of Mathematics, University of Montana, April 1977; Discrete Math. (to appear).

Department of Mathematics, University of Montana, Missoula, Montana 59812 